\title{
Multicolor fluorescence technique to detect apoptotic cells in advanced coronary atherosclerotic plaques
}

\author{
C. Soldani, A.I. Scovassi, ${ }^{*}$ U. Canosi, ${ }^{* *}$ E. Bramucci, ${ }^{* *}$ D. Ardissino,, E. Arbustini ${ }^{\S}$ \\ Dipartimento Biologia Animale and "Istituto di Genetica Molecolare, CNR, Pavia; ${ }^{* *}$ Divisione di \\ Cardiologia, IRCCS, San Matteo, Pavia, "Divisione di Cardiologia, Ospedale Maggiore di Parma, ${ }^{\text {s } I s t i t u t o ~}$ \\ di Anatomia Patologica, IRCCS, San Matteo, Pavia, Italy.
}

(C)2005, European Journal of Histochemistry

Apoptosis occurring in atherosclerotic lesions has been suggested to be involved in the evolution and the structural stability of the plaques. It is still a matter of debate whether apoptosis mainly involves vascular smooth muscle cells (vSMCs) in the fibrous tissue or inflammatory (namely foam) cells, thus preferentially affecting the cell-poor lipid core of the atherosclerotic plaques. The aim of the present investigation was to detect the presence of apoptotic cells and to estimate their percentage in a series of atherosclerotic plaques obtained either by autopsy or during surgical atherectomy. Apoptotic cells were identified on paraffinembedded sections on the basis of cell nuclear morphology after DNA staining and/or by cytochemical reactions (TUNEL assay, immunodetection of the proteolytic poly (ADP-ribose) polymerase-1 [PARP-1] fragment); biochemical procedures (identifying DNA fragmentation or PARP-1 proteolysis) were also used. Indirect immunofluorescence techniques were performed to label specific antigens for either vSMCs or macrophages (i.e., the cells which are most likely prone to apoptosis in atherosclerotic lesions): the proper selection of fluorochrome labeling allowed the simultaneous detection of the cell phenotype and the apoptotic characteristics, by multicolor fluorescence techniques. Apoptotic cells proved to be less than $5 \%$ of the whole cell population, in atherosclerotic plaque sections: this is, in fact, a too low cell fraction to be detected by widely used biochemical methods, such as agarose gel electrophoresis of low-molecular-weight DNA or Western-blot analysis of PARP-1 degradation. Most apoptotic cells were of macrophage origin, and clustered in the tunica media, near or within the lipid-rich core; only a few TUNEL-positive cells were labeled for antigens specific for vSMCs. These results confirm that, among the cell populations in atherosclerotic plaques, macrophage foam-cells are preferentially involved in apoptosis. Their death may decrease the cell number in the lipid core and generate a possibly defective apoptotic clearance: the resulting release of matrix-degrading enzymes could contribute to weakening the fibrous cap and promote the plaque rupture with the risk of acute ischemic events, while increasing the thrombogenic pultaceous pool of the plaque core.

Key words: apoptosis, atherosclerosis, macrophages, multicolor fluorescence, smooth muscle cells.

Correspondence to: Cristiana Soldani

Laboratorio di Biologia Cellulare, Dipartimento di Biologia Animale, Università di Pavia

Piazza Botta, 1027100 Pavia (Italy)

E-mail: soldani@unipv.it
A therosclerosis begins when monocytes migrate from the bloodstream into the wall of the artery and change into cells that accumulate fatty materials (Ross, 1993). This fat-laden monocyte accumulation leads to a patchy thickening in the inner lining (tunica intima) of the artery. Each area of thickening (called atherosclerotic plaque or atheroma) is filled with a pultaceous substance consisting of various fatty materials (principally cholesterol) surrounded by a fibrous cap. This fibrous tissue may be either hyperplastic and rich in vascular smooth muscle cells (vSMCs), or fibrosclerotic with dense fibrous components, and it is often infiltrated by inflammatory cells among which macrophages, $\mathrm{CD} 68^{+}$foam and non-foam cells, and CD45RO+ T-lymphocytes (Mallat and Tedgui, 2001). Atheromas may be scattered throughout the medium and large arteries, but usually they are generated where the arteries branch off, presumably because the constant turbulence at these areas injures the arterial walls, making them more susceptible to atheroma formation (Bennett, 1999).

Numerous investigations indicate that apoptosis is essential in atherogenesis, as a factor that modulates its early progression (Haunstetter and Izumo, 1998; Best et al., 1999). Changes in the rate of apoptosis contribute to differences in the growth rate, structure and stability of the plaque, depending on the stage of plaque development (Kockx and Herman, 2000) and, likely, on the cell type involved.

Apoptosis is a form of cell death characterized by a series of morphological events which can be detected by light and electron microscopy; it is now unanimously accepted that -in the great majority of cases- the morphological apoptotic changes are dependent on the concerted action of proteolytic enzymes called caspases (Chang and Yang, 2000; Salvesen, 2002) which cleave a wide spectrum of cytoplasmic and nuclear substrates (Shi, 2002; Donepudi and Grutter, 2002) and are responsible for the classical apoptotic processes of cell shrink- 
age, membrane blebbing, condensation of nuclear chromatin, nuclear and cellular fragmentation, and final engulfment of apoptotic bodies by neighboring cells (Chionna et al., 2003).

The apoptotic nuclear changes depend on the activation of endogenous nucleases (for a recent review, see Scovassi and Torriglia, 2003) which induce the cleavage of DNA, first into high-molecular-weight molecules (of about 300 to 50 kilobases) and finally into oligonucleosome-long fragments that give rise to the typical ladder in conventional agarose gel electrophoresis. The apoptotically fragmented DNA may be detected in tissue sections by the TUNEL procedure which allows the addition of labeled DNA precursors to the free $3^{\prime}$-end of cleaved DNA molecules by exogenous terminaldeoxynucleotidyl-transferase ( $\mathrm{TdT}$ ). Another biochemical feature of apoptotic cell death is the proteolysis of poly(ADP-ribose) polymerase-1 (PARP$1)$, a nuclear enzyme involved in DNA repair and activated during apoptosis in response to DNA damage; the derived $89 \mathrm{kDa}$ fragment can be detected by specific antibodies both in Western blot and in tissue sections (Soldani and Scovassi, 2002).

In the present investigation, we aimed at detecting apoptotic cells in coronary atherosclerotic plaques and defining their origin (from either vSMCs or macrophages) by immunolabeling techniques. A microscopical and histochemical approach was selected as it allows detecting in situ rare events occurring within heterogeneous cell populations (for a recent review on subject, see Mazzini et al., 2003). DNA electrophoresis and Western blot analysis for PARP-1 cleavage were also made on cellular extracts from frozen samples.

\section{Materials and Methods}

\section{Arterial specimens}

Atherosclerotic plaques were taken from coronary arteries with atherosclerosis by autopsy or during surgical atherectomy (IRCCS Policlinico San Matteo, Pavia, Italy).

The arterial specimens were either immediately stored in liquid nitrogen or immersed in formalin $4 \%$ and kept in fixative overnight at $4^{\circ} \mathrm{C}$. After dehydration, the samples were embedded in paraplast; $7 \mu \mathrm{m}$ thick serial sections were cut and either stained with haematoxilin and eosin, for the morphological analysis, or submitted to different histo- chemical procedures as reported below.

A total of 10 atherosclerotic plaques were examined.

Tunel procedure for the detection of apoptotic cells

The sections were dewaxed, rehydrated and incubated with proteinase $\mathrm{K}(20 \mu \mathrm{g} / \mathrm{mL})$ for $7 \mathrm{~min}$. at room temperature (r.t.). After washing with phosphate buffer saline (PBS), they were incubated with $3 \% \mathrm{H}_{2} \mathrm{O}_{2}$ in $10 \%$ methanol in PBS for $7 \mathrm{~min}$. to block endogenous peroxidases, then washed with PBS and incubated with $75 \mu$ of equilibration buffer for $1 \mathrm{~min}$ and with $54 \mu \mathrm{l}$ of working strength $\mathrm{TdT}$ enzyme at $37^{\circ} \mathrm{C}$ for $\mathrm{lh}$, according to the procedure suggested by the supplier (ApopTag Plus Peroxidase In Situ Apoptosis Detection Kit, Oncor). Then the sections were washed with working strength stop/wash buffer for $10 \mathrm{~min}$.at r.t. and with PBS for 15 min. After this step, some sections were incubated with $55 \mu \mathrm{l}$ of anti-digoxigenin-peroxidase for $30 \mathrm{~min}$ at r.t., and finally revealed by $0.05 \%$ $3,3^{\prime}$ diaminobenzidine tetrahydrochloride with $0.01 \% \mathrm{H}_{2} \mathrm{O}_{2}$ in $0.05 \mathrm{M}$ Tris/HCl buffer, $\mathrm{pH} 7.6$, and counterstained with haematoxilin. Parallel samples were incubated with Texas Red-conjugated antidigoxigenin (1:100), for $1 \mathrm{~h}$ at r.t. and counterstained for DNA with Hoechst $33258(0.1 \mathrm{\mu g} / \mathrm{mL})$. All the stained specimens were finally mounted with Mowiol (Calbiochem, Darmstadt, Germany).

\section{Immunohistochemical techniques}

The antibodies used to label the VSMCs were: the mouse monoclonal antibody HHF35, diluted 1:50 (Dako) to identify vSMC-specific actin. To label macrophage cells either a monoclonal antimacrophage antibody, clone LN-5, diluted 1:400 (Sigma), or a mouse anti-human CD68, clone KP1, diluted 1:100 (Dako) were used. The immunolabeled samples were finally counterstained with Hoechst 33258 and mounted as reported above.

\section{Fluorescence microscopy and digital photomicrog- raphy}

After the different cytochemical procedures, the slides were observed in fluorescence microscopy with an Olympus BX50 microscope equipped with a $100 \mathrm{~W}$ mercury lamp. The following conditions were used: 330-385 nm excitation filter (excf), 400 $\mathrm{nm}$ dichroic mirror $(\mathrm{dm})$, and $420 \mathrm{~nm}$ barrier filter (bf), for Hoechst 33258; 450-480 nm excf, $500 \mathrm{~nm}$ $\mathrm{dm}$, and $515 \mathrm{~nm}$ bf for FITC; and $540 \mathrm{~nm}$ excf, 580 $\mathrm{nm} \mathrm{dm}$, and $620 \mathrm{~nm}$ bf for Alexa 594 and Texas 
Red. Images were recorded with an Olympus Camedia C-2000 Z digital camera and stored on a $P C$ by the Olympus software, for processing and printing.

\section{Apoptotic index}

Double stained slides were used for quantification of apoptosis: the samples were processed for TUNEL reaction, revealed with $D A B$, and counterstained with haematoxilin, as reported above. At least 300 total cells were counted in 25 randomly chosen high-power fields using an eyepiece reticule with a $0.04 \mathrm{~mm}^{2}$ grid.

\section{Agarose gel electrophoresis of DNA}

To assess the presence of the apoptotic DNA fragmentation, genomic DNA was rapidly extracted from frozen atherosclerotic plaques and analyzed by agarose gel electrophoresis according to the procedure reported by Donzelli et al., (1999).

\section{SDS-PAGE and Western Blot analysis}

Protein extraction and electrophoresis were performed according to Donzelli et al., (1999). The mAb C-2-10 against PARP-1 (Alexis, VinciBiochem, Italy) was diluted 1:1000. An antimouse IgG antibody conjugated to horseradish peroxidase (HRP, Sigma) was used. Visualization of immunoreactive bands was obtained by an ECL detection system (Pierce).

\section{Results}

The procedure of specimen collection, fixation and embedding proved to be appropriate, based on the micrographs obtained after haematoxilin and eosin staining (figure $1 \mathrm{a}, \mathrm{c}$ ). The cross sections of the coronary arteries showed a pronounced morphological variability in the different wall regions, due to the presence of the atherosclerotic lesions: in the tunica media, the density of nuclei was lower
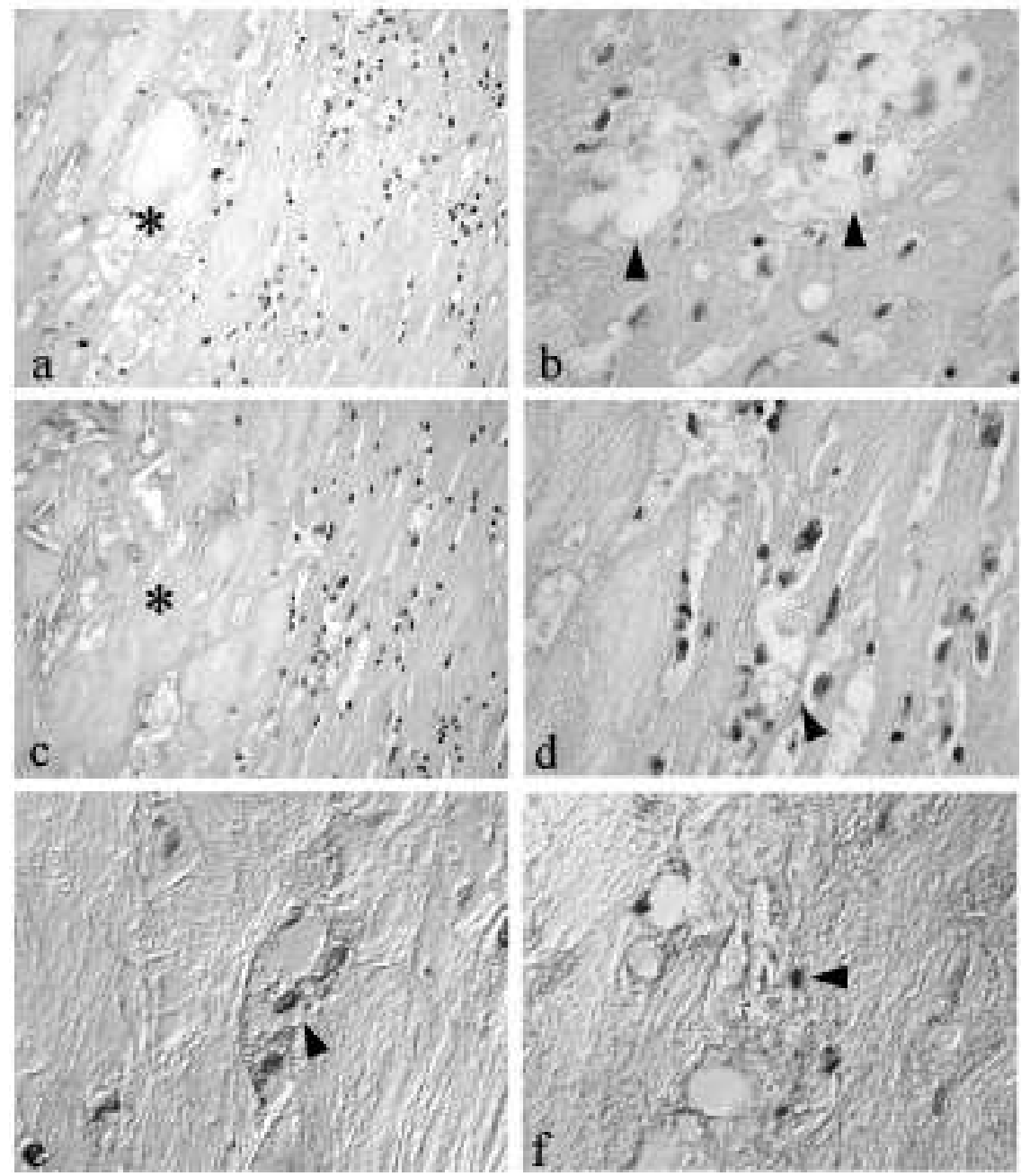

Figure 1. Haematoxilin and eosin on sagittal sections of atherosclerotic plaques (a-d). At lower magnification $(10 x$, a and c) the variability of the morphology and cell distribution in the different regions was visible: in the tunica media, the density of nuclei was less then the intima and adventitia (Figures a,c, asterisks). The cells in this area, called foam cells, showed lipids located within the cytoplasm and the darkerstaining nuclei (Figures b,d arrowheads). TUNEL assay on the plaques (e-f): the TUNEL-positive cells were mainly located in the tunica media and had the characteristic foam morphology (Figures ,e,f, arrows heads). 

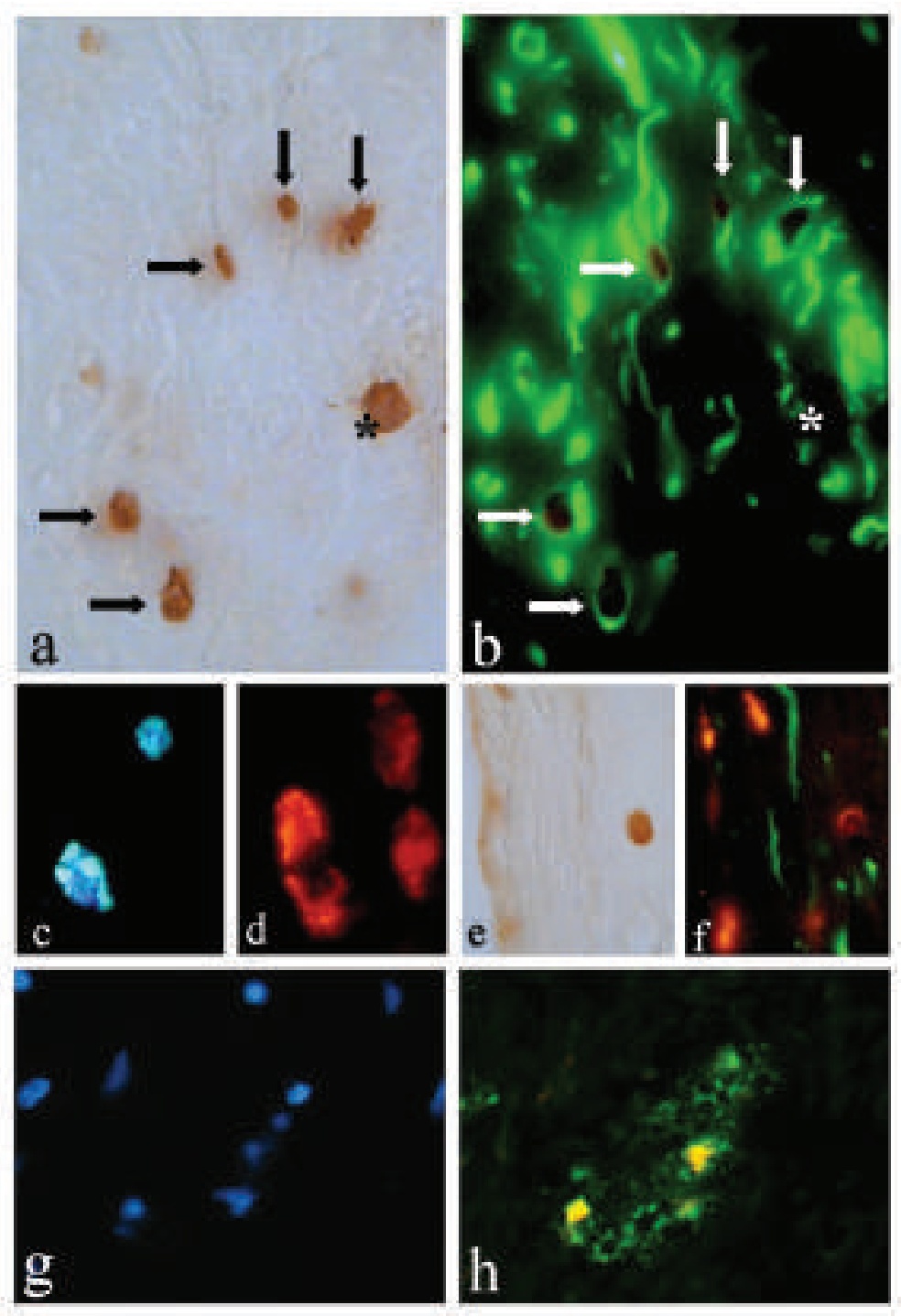

Figure 2. Multicolor fluorescence on the atherosclerotic plaques (a-h). Immunostaining with HHF35 showed that several TUNEL-positive nuclei, belonged to vSMCs (a,b, arrows); in the same figure, an apoptotic nucleus (asterisk) was negative for the cytoplasmic HHF35 staining. Two cells with nuclear apoptotic morphology (c) were labeled with the anti-macrophage antibody (red fluorescence, d), and this was confirmed by experiments of simultaneous TUNEL procedure and two-color fluorescence immunolabeling for vSMC-specific actin (green fluorescence) and CD68 (red fluorescence: Figures e,f). Apoptotic foam cells of macrophage origin were mostly located in the tunica media (Figures g,h).

than in the intima and adventitia (Figures $1 a, c$, asterisks). The cells in this area, called foam cells, showed lipids located within the cytoplasm and darker-staining nuclei (Figures 1b,d arrowheads).

The occurrence of apoptotic DNA fragmentation was detected by the TUNEL assay (Figures le,f). TUNEL-positive cells with typically condensed nuclei were mainly located in the tunica media (Figures $1 e, f$ ) and had the characteristic foam morphology (Figures le,f, arrowheads), but were also present in smaller amount in the sub-intimal layer. The total apoptotic index ranged between 3\% and $4 \%$.

To identify the tissue origin of apoptotic cells, multicolor fluorescence techniques were used. In Figures 2 ( $a, b$, arrows), the immunostaining with HHF35 showed that in the intimal and subintimal layer a few TUNEL-positive nuclei, belong to
vSMCs; in the same figure, however, an apoptotic nucleus (asterisk) was negative for the cytoplasmic HHF35 staining. In fact, several cells of macrophage origin also proved to be apoptotic, especially in the tunica media: in Figures 2c, d, two cells with nuclear apoptotic morphology were labeled with the anti-macrophage antibody (red fluorescence), and this was confirmed by experiments of simultaneous TUNEL procedure and two-color fluorescence immunolabeling for vSMC-specific actin (green fluorescence) and CD68 (red fluorescence: Figures 2e,f). Apoptotic foam cells of macrophage origin were mostly located in the tunica media (Figures $2 \mathrm{~g}, \mathrm{~h}$ ).

Due to the low percentage of apoptotic cells, biochemical assay routinely used to detect the presence of apoptotic cells, i.e., agarose gel electrophoresis of low-molecular-weight DNA and 

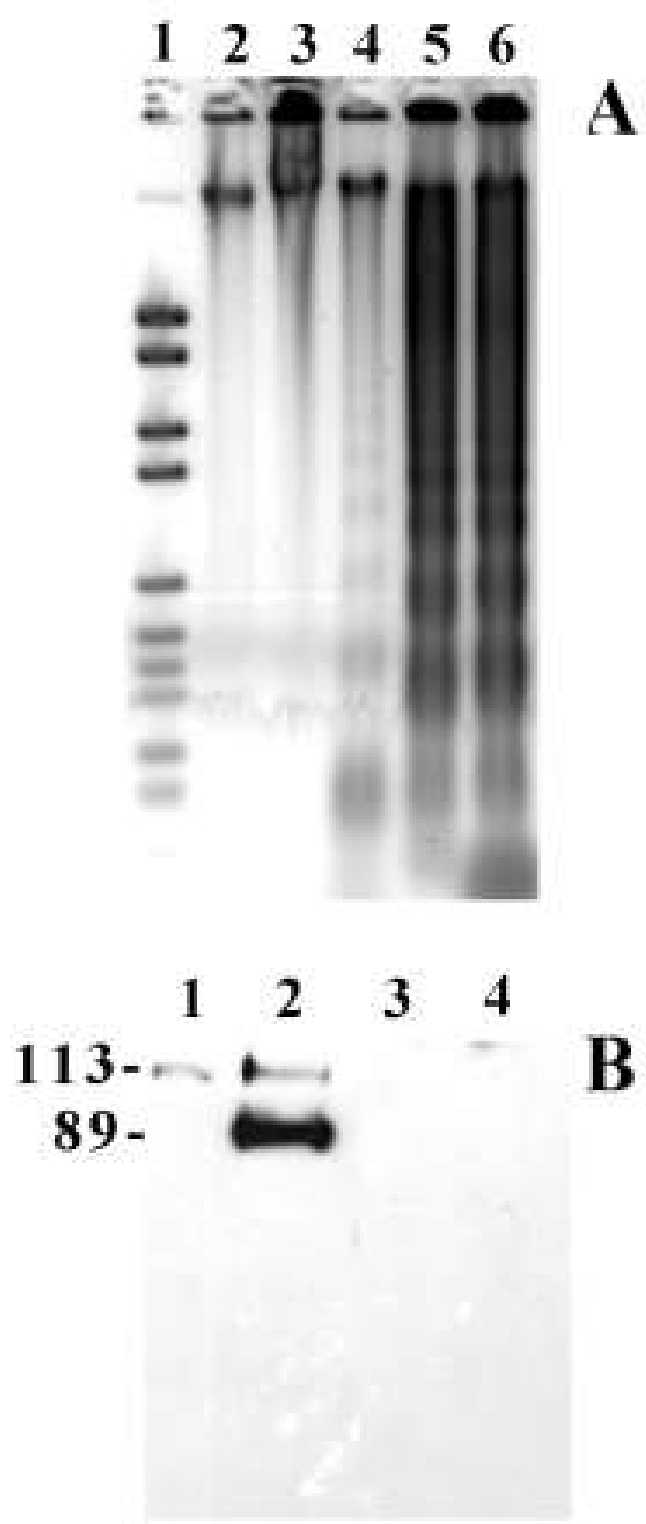

Figure 3. DNA and protein degradation in apoptotic samples. A) Agarose gel electrophoresis for low-molecular-weight fragments. Lane 1: molecular markers; lane 2,3: atherosclerotic plaques; lane4: mouse apoptotic thymus; lane 5,6: apoptotic Hela cells. B) Western blot for PARP-1 cleavage. Lane 1: control Hela cells; lane 2: apoptotic Hela cells; lane 3,4: atherosclerotic plaques. 113 kDa: intact PARP-1; 89 kDa: cleaved fragment.

Western-blotting analysis of PARP-1 cleavage, failed to give positive results with the material from atherosclerotic plaques (Figure 3 ).

\section{Discussion}

Our histochemical results demonstrate that apoptotic cells comprise less than $4 \%$ of total cells in the sections. This low apoptotic index accounts for the inability to reveal the presence of apoptosis by the electrophoretic analyses of DNA fragmentation or PARP-1 cleavage which, in our experience, give positive results when the percentage of apoptotic cells is higher than 10\% (Negri et al., 1997).

The cytochemical approach proved to be unique, as it allows identification of the relatively rare apoptotic events occurring in the atherosclerotic plaques; moreover, it is worth considering that cytochemical techniques may be conveniently used on routinely archived material.

The cellular composition of an atherosclerotic plaque is the major determinant of its stability; in particular, plaques rich in cholesterol-loaded macrophages and in extracellular lipid deposits are generally unstable and highly vulnerable for rupture, which is the major cause of the fatal effects of atherosclerosis.

The occurrence of apoptosis in atherosclerotic plaques may have a double pathophysiological meaning: on the one hand, apoptotic cell death may influence plaque progression (thus being a self-limiting factor in plaque development) and may also quench the inflammatory reaction without affecting plaque volume (Kolodgie et al., 1999); on the other hand, cell death might promote the conversion of a cellular-rich lesion to a fibrous hypocellular (so-called stable) plaque.

Consistently, apoptosis involving VSMCs could counterbalance their proliferation and thus reduce the rate of plaque growth. The loss of macrophages could also lead to plaque stabilization, since they can promote local expression and/or activation of matrix metalloproteinases, which decrease the strength of the fibrous cap by degrading interstitial collagen fibers (Galis et al., 1994, 1995); apoptosis of macrophages then can reduce both proteolytic activity and the production of pro-apoptotic signals inside the lesion (Guevara et al., 2001).

However, vSMCs are also the major source of interstitial collagen fibers (Rekhter, 1999) which play an important structural role in atherosclerotic lesions: the loss of VSMCs could lead to a depletion of collagen-I fibers, thus critically affecting plaque stability (Rekhter, 1999) and possibly increasing its thrombogenicity (Flynn et al., 1997). Similarly, a loss of macrophage cells would result in decreased scavenging of the products of cell degradation, leading to the accumulation of necrotic debris within the plaque, which may destabilize the lesion and promote thrombin activation (Kockx and Herman, 
2000; Kockx and Knaapen, 2000).

The results of the present investigation proved that apoptosis mainly involves cells of macrophage origin (the foam cells in the tunica media), but may also occur in cells of smooth muscle origin (in the sub-intimal layer). The simultaneous application of histochemical techniques for detecting apoptotic features and cell differentiation markers promises to be a powerful tool in the elucidation of the role of different tissue components in the generation of the atherosclerotic lesion. When applied to samples obtained from patients by atherectomy, these techniques should also provide information on the evolution of plaque stability and be helpful in selecting the most suitable therapeutic options.

\section{Acknowledgements}

We are grateful to Prof. Carlo Pellicciari for his helpful discussion. Research was supported by the University of Pavia (Progetto Giovani Ricercatori) and by MIUR (FIRB project RBNE0132MY).

\section{References}

Bennett MR. Apoptosis of vascular smooth muscle cells in vascular remodeling and atherosclerotic plaque rupture. Cardiovasc Res 1999; 41:361-8.

Best PJ, Hasdai D, Sangiorgi G, Schwartz RS, Holmes DR, Simari RD, Lerman A, Apoptosis. Basic concepts and implications in coronary artery disease. Arterioscler Thromb Vasc Biol 1999; 19:14-22.

Chang HY, Yang $X$. Proteases for cell suicide: functions and regulation of caspases. Microbiol Mol Biol Rev 2000; 64:821-46.

Chionna A, Panzarini E, Pagliara ., De Luca A, Caforio S, Abbro L, et al. Hepatic clearance of apoptotic lymphocytes: simply removal of waste cells? Eur J Histochem 2003; 47:97-104.

Donepudi M, Grutter MG. Structure and zymogen activation of caspases. Biophys Chem 2002; 101-102:145-53.

Donzelli M, Bernardi R, Negri C, Prosperi E, Padovan L, Lavialle C, et al. Apoptosis-prone phenotype of human colon carcinoma cells with a high level amplification of the c-myc gene. Oncogene 1999; 18:439-48.

Flynn MA, Herbert V, Nolph GB, Krause G. Atherogenesis and the homocysteine-folate-cobalamin triad: do we need standardized analyses? J Am Coll Nutr 1997; 16:258-67.

Galis ZS, Sukhova GK, Lark MW, Libby P. Increased expression of matrix metalloproteinases and matrix degrading activity in vulnerable regions of human atherosclerotic plaques. J Clin Invest 1994; 94:2493-503.

Galis ZS, Sukhova GK, Kranzhofer R, Clark S, Libby P. Macrophage foam cells from experimental atheroma constitutively produce matrix-degrading proteinases. Proc Natl Acad Sci USA 1995; 92:402-06.

Guevara NV, Chen KH, Chan L. Apoptosis in atherosclerosis: pathological and pharmacological implications. Pharmacol Res 2001; 44:59-71.

Haunstetter A, Izumo S. Apoptosis: basic mechanisms and implications for cardiovascular disease. Circ Res 1998; 82:1111-29.

Kockx MM, Herman AG. Apoptosis in atherosclerosis: beneficial or detrimental? Cardiovasc Res 2000; 45:736-46.

Kockx MM, Knaapen MW. The role of apoptosis in vascular disease. J Pathol 2000; 190: 267-80.
Kolodgie FD, Narula J, Guillo P, Virmani R. Apoptosis in human atherosclerotic plaques. Apoptosis 1999; 4:5-10.

Mallat Z, Tedgui A. Current perspective on the role of apoptosis in atherothrombotic disease. Circ Res 2001; 88:998-1003.

Mazzini G., Ferrari C., Erba E. Dual excitation multi-fluorescence flow cytometry for detailed analyses of viability and apoptotic cell transition. Eur J Histochem 2003; 47:289-298

Negri C, Donzelli M, Bernardi R, Rossi L, Burkle A, Scovassi AI. Multiparametric staining to identify apoptotic human cells. Exp Cell Res 1997; 234:174-77.

Rekhter MD. Collagen synthesis in atherosclerosis: too much and not enough. Cardiovasc Res 1999; 41:376-84.

Ross R. The pathogenesis of atherosclerosis: a perspective for the 1990s. Nature 1993; 362:801-9.

Salvesen GS. Caspases and apoptosis. Essays Biochem 2002; 38:9-19.

Scovassi AI, Torriglia A. Activation of DNA-degrading enzymes during apoptosis. Eur J Histochem 2003; 47:185-94.

Shi Y. Mechanisms of caspase activation and inhibition during apoptosis. Mol Cell 2002; 9:459-70.

Soldani C, Scovassi AI. Poly(ADP-ribose) polymerase-1 cleavage during apoptosis: an update. Apoptosis 2002; 7:321-8. 